\title{
Parlamento abierto y democracia: un debate sobre lo más allá de la poliarquía
}

\author{
Freddy Mariñez Navarro ${ }^{1}$, \\ El Colegio de Jalisco, Zapopan - México
}

\begin{abstract}
Recebido em 5 de Agosto de 2016. Aceito em 29 de Agosto de 2016 RESUMO

Este trabalho tem por objetivo analisar a relação entre o parlamento aberto com o governo aberto no contexto de ferramentas democráticas além da poliarquia. O trabalho se desennvolve em quarto pontos com o objetivo de ir atriculando estes três conceitos. Em primeiro lugar, é introduzido no contexto de um governo aberto; depois, em um segundo momento se aborda a questão do parlamento aberto como uma expressão do governo aberto. E, finalmente, analizamo-se os saberes cívicos como uma ferramenta política além da poliarquia.
\end{abstract}

Palavras-chave: além da poliarquia, governo aberto, parlamento aberto, saber cívico. ABSTRACT

This paper aims to analyze the relationship between parliament opened with the open government in the context of democratic tools plus poliarchy. It will involve three points with the idea of going articulating these three concepts. First, the context of open government is introduced; after we boarded a second time parliament opened as an expression of that. And finally explain civic wisdom as a political tool plus poliarchy.

Keywords: polyarchy plus, open government, open parliament, civic wisdom. RESUMEN

Este artículo tiene como objetivo analizar la relación entre el parlamento abierto con el gobierno abierto en el contexto de las herramientas democráticas de la poliarquía plus. Se desarrollará el trabajo en cuatro puntos con la idea de ir articulando estos tres conceptos. Primeramente se introduce el contexto del gobierno abierto; luego abordamos en un segundo momento el parlamento abierto como expresión de aquel. En la tercera parte explicamos los aspectos clave de las instituciones de la poliarquía plus. Y para finalizar analizamos los saberes cívicos como una herramienta política de la democracia con poliarquía plus.

Palabras clave: poliarquía plus, gobierno abierto, parlamento abierto, compromiso cívico

\section{El gobierno abierto como contexto}

Las distintas definiciones de gobierno abierto tales como Collaborative Public Management (McGuire 2009), Citizen Engagement (Kickle, 2008; OECD, 2004), Wiki Government (Noveck, 2009) y Public Sector Clients (Alford; 2009); tienden a identificar, evaluar y caracterizar diferentes formas de integración ciudadana en el sector público tanto por la vía de la transparencia, el acceso a la información y la rendición de cuentas, así como por la colaboración, la participación y la co-creación. En este punto, retomamos el concepto inicial de gobierno abierto compuesto por sus tres componentes: transparencia, colaboración y participación (Obama, 2009).

De esta manera, para el gobierno abierto tanto su dimensión relacional (participación y colaboración) como su dimensión de apertura (transparencia, rendición de cuentas y apertura a la información), la esfera pública está construida alrededor del sistema de comunicación social y de redes de

\footnotetext{
${ }^{1}$ Profesor-Investigador de El Colegio de Jalisco.

e-mail: freddy.marinez@coljal.edu.mx
} 
internet, particularmente en el espacio social de la Web 2.0 (Open data, datos disponibles, Open Collaboration, Open Knowledge, ciudadanía 2.0, free software o social -Facebook, MySpace-, Open Source, Big data, linked data), que genera colaboración, interacción e inteligencia cívica, utilizando las aplicaciones que los usuarios pueden pueden usar para entrar al internet o intranet (webmail, wikis, weblogs, wikis, wikipedia). Ello ha implicado un cambio del proceso, modo y patrón de gobierno, de su relación con la sociedad, del paso de la cooperación a la colaboración y la co-creación, así como del tipo de transparencia colaborativa. Ya hoy el Gobierno Abierto se rige por seis principios clave: transparencia, participación, colaboración, rendición de cuentas, co-creación y TICs.

El concepto de gobierno abierto nos remite a un tipo de organización relacional como opuesta a la organización cerrada de estilo weberiana (Weber 2013; Raelin 2011; du Gay 2000). Hoy las organizaciones y, particularmente la organización pública, están en presencia de cambios significativos. Si el gobierno, tanto en términos organizativos como funcionales, lo podemos ver en su dimensión legal, ateniéndose a lo establecido en las leyes y reglamentos, que se erige como un conjunto de instituciones que pueden ser consideradas las "reglas de juego" que establecen diferentes divisiones de poderes y relaciones entre órganos unipersonales y multipersonales (Colomer; 2009), y donde descansa la legitimidad y la legalidad del poder administrativo en los principios esenciales como la eficiencia, la eficacia y los intereses generales; entonces el gobierno abierto podría entenderse como un cambio relacional y organizacional que ha implicado en su análisis y puesta en marcha innovación no sólo en los procesos, técnica y administrativamente, sino en la relación con la sociedad que ha impactado en los principios burocráticos tales como el ejercicio continuo, la sujeción a la ley de funciones dentro de una competencia, significando ésta un ámbito de deberes y servicios objetivamente limitado en virtud de una distribución de funciones; con la distribución de poderes necesario para su realización; y con la fijación estricta de los medios coactivos administrativos.

Bajo lo anteriormente planteado, un problema adicional entonces se presenta en los gobiernos actuales y es el tratamiento racional del control de las burocracias. Hoy vemos que éstas están reconfigurándose cada vez más. Bajo las presiones de la globalización, la personalización en masa y la hipercompetencia, las grandes organizaciones se han convertido en organizaciones flexibles y en muchos casos virtuales y, como los límites del sistema se han vuelto más porosos, muchas de éstas participan en redes de asociaciones. "Los formatos posburocráticos se han propuesto para producir una mayor influencia compartida, el consenso y la confianza en el interior de toda la organización, obviando la necesidad de la jerarquía y la conducta gobernada por reglas rígidas" (Sewell, 1998, citado por Raelin, 2010).

Gobierno abierto en su dimensión relacional. Desde los primeros debates, gobierno abierto presenta una dimensión relacional expresada por dos elementos, una, la colaboración a través de las tecnologías de Información y Comunicación y, la otra, la participación. Mientras la colaboración se manifiesta en el momento en que los diferentes actores intercambian conocimientos y experiencias, saberes y capital social, la participación se ubica en el marco de la distribución del poder.

Así entonces, la participación colaborativa se da en el momento en que los individuos son parte integrante, con su expertise, junto con el gobierno en el proceso de la hechura de las decisiones para generar soluciones que luego serán implementadas. Ello implica un cambio en el juego de las legitimidades tanto políticas, técnicas y sociales de los actores no gubernamentales, además de las transformaciones en las organizaciones públicas presionadas por ambientes cada día más complejos (Rainey, 2003). Tanto en las iniciativas que provienen de la ciudadanía y que en sus procesos integra a los ciudadanos como co-productores, así como en las relaciones entre el sector privado con el gobierno por vía de las Asociaciones Pública-Privadas (APPs), y las relaciones intragubernamentales, el uso de las TICs digitales posibilita este intercambio de experiencias y conocimientos para la solución de problemas. En otras palabras, la colaboración abierta ofrece una posibilidad potencial para construir de forma más eficaz y legítima los gobiernos, mediante la co-creación de lo público. 
En las actuales circunstancias y en el marco del gobierno abierto, dicen O'Leary and Vij (2012), se necesitan gerentes públicos que trabajen colaborativamente, facilitando y operando arreglos multiorganizacionales para resolver problemas que no pueden resolverse, o fácilmente resolverse, por una sola organización. Lo anteriormente afirmado por O’Leary y Vij ha sido producto de algunos cambios en el ambiente de lo público, lo privado y en las organizaciones de la sociedad civil y sin fines de lucro, que a su vez han motivado el crecimiento de lo público colaborativo.

Retomando, podemos afirmar que las aplicaciones Web 2.0 han puesto un avance no sólo en los términos tecnológicos sino también en los nuevos modos de interacción que ha generado un espacio digital donde emergen nuevas maneras de relacionarse entre las personas, las instituciones y las organizaciones. Ese espacio digital se manifiesta en las organizaciones públicas y ciudadanas con las siguientes características:

a) Abiertas y flexibles que permita potenciar la inteligencia cívica que no es más que tener información y comprenderla para compartirla a quienes necesitan respuestas. En otras palabras, implica conocer, responder y proveer, por lo que se podría entender como la capacidad de las organizaciones ciudadanas y comunitarias de adquirir y aplicar conocimientos.

b) Trabajo en red. Éste es una forma distinta de hacer las cosas, que supone ir construyendo relaciones, aprendizajes, complicidades con la idea de co-crear y/o co-producir las informaciones, las ideas, las estrategias y las formas de hacer las cosas.

c) Liderazgo relacional. En experiencias organizativas públicas, todos son líderes porque todos aportan información para la participación colaborativa. Es un liderazgo horizontal y colaborativo.

d) Innovadoras por el uso de las TICs y también por generar nuevos procesos y organización para un nuevo servicio público. Debemos centrarnos en la transformación de los modelos organizativos y en su forma de gestionarlos, de forma que generen nuevo valor para la sociedad. La innovación por la vía de modelos organizativos y formas de gestionarlos para un servicio generador de valor público.

De esta manera, tal como lo precisa Cusba (2012), la colaboración es entonces uno de los elementos que forman parte de ese gran cambio que se produce en las formas de gestionar los intereses públicos. Tradicionalmente, los ciudadanos han colaborado a través de mecanismos diferentes, unos impuestos y otros más espontáneos. Pero la colaboración vista a la luz del gobierno abierto ofrece a los ciudadanos la posibilidad de compartir lo que saben y sobre todo de crear soluciones que generan valor en donde realmente quieren y pueden aportar.

Gobierno abierto en su dimensión de Apertura. La otra dimensión del Gobierno Abierto es la relacionada con el acceso a la información pública y la transparencia. Ello implica, por un lado, datos disponibles o accesibles para cualquier persona u organización sin ninguna restricción de uso (patentes, derecho de autor, copyright y otras formas de protección legal). Y por otro lado, el gobierno abierto debe partir del Open Data como una práctica que surge como resultado de la emergencia de varias ideas o prácticas, la mayoría proveniente de las TICs (Software libre, Open Source, Big Data -datos masivos- y Linked Data -datos vinculados-).

La literatura de la transparencia en su relación gobiernos y ciudadanos ha enfatizado fuertemente el concepto de apertura: Trasparencia se define generalmente como el flujo abierto de información (Holzner, 2006). Es por esto que hoy se están encontrando nuevas pistas a la definición de ella. La apertura del flujo de información, tal como se le define, en el marco de la relación gobierno y ciudadanos nos sumerge al concepto de la nueva transparencia que explica cómo las organizaciones públicas y actores gubernamentales se enfrentan cada vez más a las demandas de la sociedad para revelar información. "La transparencia es la disponibilidad de la información sobre una organización o actor el cual permite a actores externos monitorear el trabajo interno o resultados de esta organización o actor" (Grimmelikhijsen and Welch, 2012; citado por Grimmelikhijsen; 2012: 53). Es decir, forzar al gobierno y al sector cívico al movimiento por una sociedad abierta. Ya no es el tiempo en que los gobiernos pasivamente proveían información solamente sobre lo solicitado y a la discreción gubernamental, sino el requisito de más compromiso activo en la información pública, comunicar las operaciones internas de la gestión. Por eso la 
relación entre transparencia, información y democracia es fundamental y básica. La información porque ayuda a las competencias democráticas como la formulación de preferencias y opiniones probadas y, la participación, porque provee elementos significativos en la hechura de la decisión pública.

"El libre acceso a la información en el poder de la Administración Pública es una condición para el ejercicio de la participación, la que sólo puede verse limitada por escasas restricciones claramente establecidas. Entre otras cosas, incluye el acceso a archivos y registros, fijación de plazos máximos de respuestas del gobierno, la recepción de la información en formatos accesibles, el conocimiento fundado de los motivos por las cuales no se suministra total o parcialmente y otras condiciones por el estilo (Oszlak; 2012: 10).

La transparencia en una organización no sólo es lo que es comunicado externamente, sino sobre cómo en el interior de la administración se hacen las cosas. La nueva transparencia es la tendencia de la organización a hacer frente a las demandas más enérgicas para la divulgación de la información. En el pasado, muchos gobiernos pasivamente proporcionaban información únicamente bajo petición y discreción. Ahora éstos están siendo obligados a participar más activamente en la divulgación de la información.

Esta demanda fuertemente creciente de apertura mediante la transparencia se basa en varios factores:

1.- La transparencia es una de las reivindicaciones morales fundamentales en las sociedades democráticas, con los derechos de los ciudadanos a tener acceso a la información gubernamental está ampliamente aceptado en las democracias representativas.

2.- La transparencia es una de las medidas prácticas tomadas para disminuir la corrupción, actuando como elemento disuasorio contra las conductas corruptas mediante la promoción de la vigilancia ciudadana, impidiendo a los funcionarios públicos lograr beneficio privado con el mal uso de los servicios público

3.- La transparencia tiene un efecto positivo sobre la confianza y la rendición de cuentas. La transparencia está ligada o conectada con los valores de la rendición de cuentas, ya que permite a los ciudadanos vigilar la calidad del servicio público y alienta a los empleados públicos para satisfacer a los ciudadanos

En este sentido, podemos definir la transparencia como una herramienta de control en manos de los ciudadanos, un medio de lucha contra la corrupción, una vía de participación ciudadana, y una manera de poner en valor datos públicos que de otro modo no se aprovecharán.

Tenemos, entonces a manera general, varios tipos de transparencia, dentro de las cuales precisamos cuatro:

a.- Transparencia pasiva (reactiva) se refiere al derecho ciudadano de acceso a la información pública y aquella que genera el gobierno. Requiere que exista un mandato legal para que las instancias gubernamentales publiquen y actualicen información.

b.- Transparencia focalizada es un modelo de transparencia "bajo demanda", donde la información es liberada por el gobierno y focalizada en áreas específicas, buscando resolver necesidades concretas.

c.- Transparencia activa (o proactiva) se define como la obligación de la administración de presentar información, sin necesidad de que la misma sea previamente solicitada.

d.- Transparencia colaborativa es un modelo de transparencia basado en el uso de las TICs y los datos abiertos (Open data) como herramientas básicas. Este modelo es de tipo interactivo y precisa de una colaboración entre organismos que permita transformar grandes volúmenes de datos. Se caracteriza por la obligación de los gobiernos y administraciones de publicar los datos "crudos" y el derecho de los ciudadanos a procesarlos para producir nueva información con ellos. 
Estos elementos del Gobierno Abierto es el fundamento contextual del Parlamento Abierto. Hoy, estamos en presencia de demandas sociales -bien de apertura, bien relacionales- que exigen ir más allá de las instituciones liberales poliárquicas para que la transparencia genere en las organizaciones una tendencia hacer frente a las demandas de la divulgación de la información, así como de la participación, la colaboración y la co-creación.

\section{Parlamento abierto como una expresión del gobierno abierto}

En el mundo democrático actual, existen limitaciones inherentes a la realidad del sistema representativo, por lo que podemos afirmar que son uno de los problemas fundamentales de las democracias contemporáneas, resumiéndose de la siguiente manera:

1.- Autonomización de los parlamentarios y los partidos políticos durante el ejercicio del mandato, con un desprecio consecuente por la opinión de los votantes, la pérdida de la relación de confianza entre el Parlamento y los ciudadanos;

2.- El dominio de los grupos económicamente más poderosos y con estructura de lobby más organizada en los parlamentos, la desconexión con el sistema legal y la falta de calidad en la preparación de las leyes, la incapacidad del Parlamento para responder a las demandas cada vez más complejas y distintas de la sociedad;

3.- La falta de ética de modo general por parte de los parlamentarios que emplean los recursos institucionales para el ejercicio del mandato de manera irregular, con faltas injustificadas a sesiones parlamentarias, desaplicación en el trabajo parlamentario, entre algunos de los comportamientos perjudiciales.

De modo más exacto, Fung (2007) sostiene que el sistema liberal presentaría cuatro dificultades que generan los déficits democráticos:

1.- los ciudadanos no tienen preferencias claras, independientemente de las políticas que se puedan adoptar, o incluso tales preferencias pueden cambiar fácilmente cuando los ciudadanos son expuestos a nuevas informaciones, argumentos y perspectivas.

2.- Cuando los ciudadanos poseen preferencias claras y estables y los mecanismos electorales proporcionan apenas señales débiles a los políticos y a los partidos sobre el contenido de estas preferencias. La falta de una conexión fuerte e incesante entre políticos y ciudadanos hace que los primeros no sepan muy bien lo que los últimos desean. Y sin ella no hay manera de obtener una buena representación.

3.- Mecanismo de elección insuficiente para propósitos de rendición de cuentas (accountability) de los políticos. En muchas decisiones de Estado los intereses de los políticos y los administradores públicos pueden diferenciarse de los intereses de la mayoría de los ciudadanos. Por lo tanto, es difícil para los votantes emplear las elecciones como instrumento de control sobre la acción de los políticos durante el ejercicio de su mandato.

4.- Aunque el sistema electoral de representación y de rendición de cuentas permitiera el control de los ciudadanos sobre sus representantes políticos y administrativos (de una manera casi ideal), el Estado no tendría la capacidad técnica para proporcionar resultados razonables de políticas que materializan las preferencias de los ciudadanos.

Visto estos déficits, podemos ver que un país que se dice ser democrático, se inhiben sus actividades sustantivas, tales como:

a.- Los representantes deberían discutir y aprobar leyes con base en las necesidades, demandas, inquietudes y experiencias de las personas a quienes representan.

b.- Dada la importancia de esta tarea, es imprescindible que los legisladores puedan realmente contar con toda la información necesaria para poder elaborar buenas leyes, que permitan alcanzar los objetivos más apreciados por los distintos grupos sociales. 
Una de las formas de ser congruente con estas actividades sustantivas, es lograrlo mediante la inclusión de grupos, colectivos, movimientos y personas que tienen conocimientos, prácticos o teóricos, sobre los temas que se busca legislar. Esto no significa que los diputados o senadores no conozcan sobre esas materias, sino que ese conocimiento debe complementarse con otras perspectivas que, en algunos casos, son más cercanas al problema que se quiere solucionar con la ley en cuestión.

El primer objetivo sustantivo de un Parlamento Abierto es establecer una nueva relación entre representantes y representados, en términos de colaboración, rendición de cuentas, acceso a la información, participación ciudadana y uso de tecnologías de información (2014). Ello con la intención de minimizar por una parte, la insatisfacción popular, y por la otra, la desconfianza en la institución parlamentaria.

En cuanto a la minimización de la insatisfacción popular, es necesario que el Parlamento Abierto tenga una dimensión relacional dinamizada a través de la colaboración y la participación entre los parlamentarios de distintos partidos políticos e independientes, las comisiones asesoras y los ciudadanos y sus organizaciones para que se logre el intercambio de experiencias, conocimientos, saberes e inteligencia cívica para hacer de las decisiones parlamentarias y de los acuerdos un acto legítimamente relacional. Es la vía para el logro de la satisfacción popular y esto se basaría en tres de los diez principios, hoy propuestos por la Alianza por el Parlamento Abierto (2014): 1- Información parlamentaria, 2.- información histórica; y 3.- Información sobre legisladores y servidores públicos.

Esta dimensión relacional hace del Parlamento Abierto una institución legislativa que pone a disposición de la sociedad información oportuna, sencilla y reutilizable, promueve la transparencia, y establece mecanismos para involucrar real y efectivamente a la ciudadanía en el trabajo legislativo, utilizando tecnologías de la información y la comunicación. De esta manera, la arena política en redes esfera del debate público-, permite el trabajo colaborativo de diversas formas. Como por ejemplo, individuos pueden monitorear y desvirtuar el poder de los medios convencionales, así como organizar acciones políticas, mediante las diversas formas de acceso a Internet, los individuos y grupos estarían más aptos a observar, relatar, comentar y analizar hechos, ejerciendo en suma, las funciones de agentes de medios con capacidad de atraer la atención pública a los diversos asuntos políticos.

Y por otro lado, al referirnos a la minimización de la desconfianza, el Parlamento Abierto hace uso de su dimensión de apertura mediante la transparencia, la rendición de cuentas y los datos abiertos para que los ciudadanos y demás actores no gubernamentales puedan disponer de la información y a su vez ver con claridad las normas del parlamento, sus reglamentos y cómo se toman sus decisiones. Esta dimensión de apertura se basa en los siguientes siete principios de la Alianza por el Parlamento Abierto: 4- Derecho a la información; 5- Participación ciudadana y rendición de cuentas 6- Información presupuestal y administrativa; 7- Datos abiertos y No Propietarios; 8- Accesibilidad y difusión; 9.- Conflicto de interés; y 10.- Legislar a favor de Gobierno Abierto. (Vease Gráfica No. 1) 


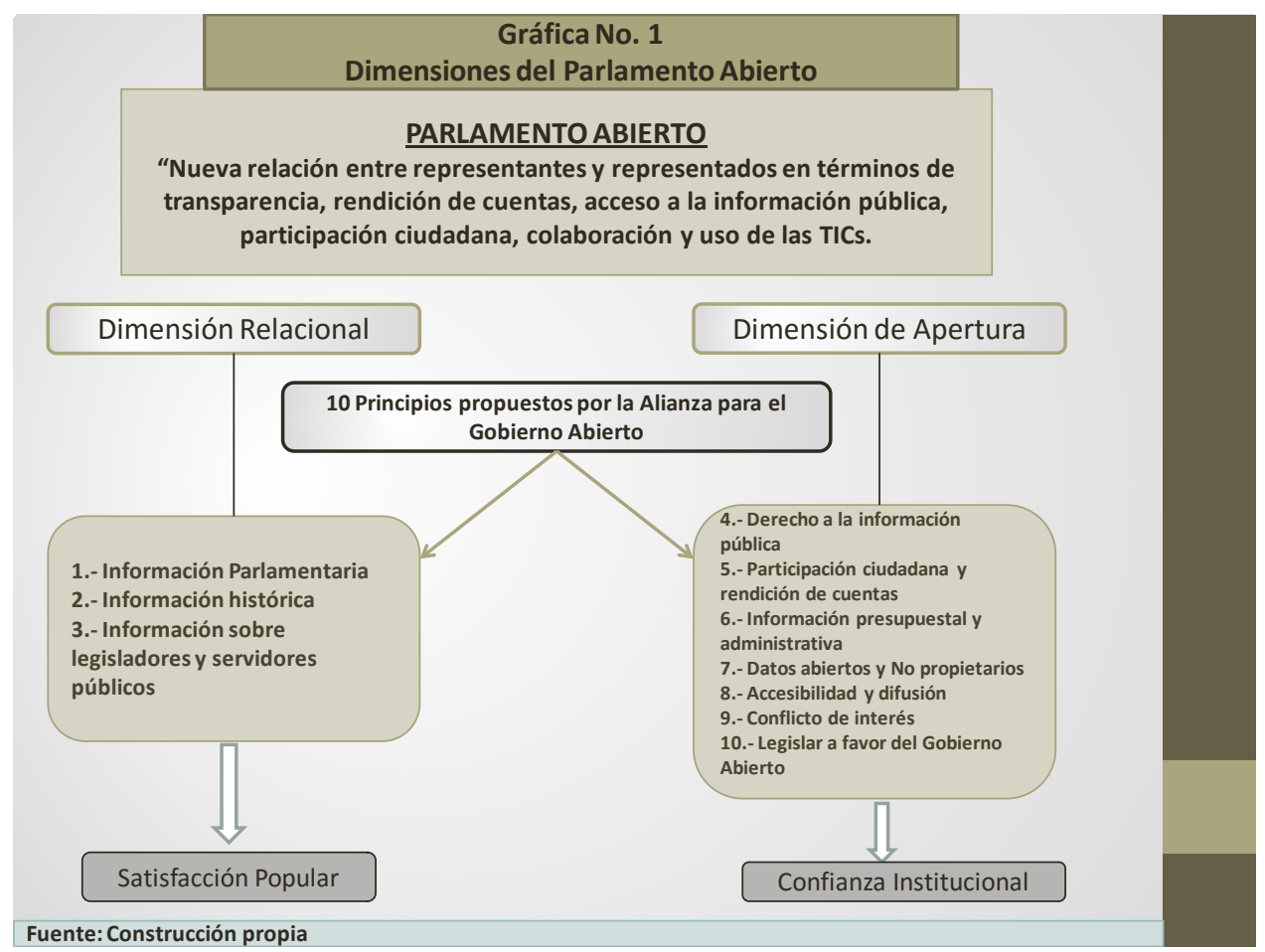

\section{Grafica 1 - Dimensiones del Parlamento Abierto}

En el caso de México, la Alianza para el Parlamento Abierto define el Parlamento abierto como un espacio de encuentro de organizaciones de la sociedad civil, el Órgano Garante de Acceso a la Información y Protección de Datos y las instituciones legislativas, cuyo objetivo es lograr que los 32 congresos locales y el Congreso de la Unión cumplan con los principios y acciones de un parlamento abierto. Doce organizaciones como Arena Ciudadana, Borde Político, Consorcio para el Diálogo Parlamentario y la Equidad, Gesoc, IMCO, Impacto Legislativo, Fundar, Centro de Análisis e Investigación, OPI, Social TIC, Sonora Ciudadana, Transparencia Mexicana y Visión Legislativa se unieron para impulsar la Alianza del Parlamento Abierto, una iniciativa que busca promover la agenda de parlamento abierto en México para establecer una nueva relación entre representantes y representados, en términos de rendición de cuentas, acceso a la información, participación ciudadana, colaboración y uso de tecnologías de información (Parlamento abierto en México, 2014).

Es importante dejar claro que para que se lleven a cabo estas dimensiones del Parlamento Abierto se requieren de algunas precondiciones, como las siguientes:

1. Políticos dispuestos a colaborar y ser controlados por los ciudadanos (dispuestos a cambiar su comportamiento habitual más allá del mínimo requerido legalmente de la transparencia y la inclusión en los procesos de toma de decisiones).

2. Ciudadanos dispuestos a colaborar y controlar con y a los políticos.

3. Iniciativa de Innovación Pública por parte del Poder Legislativo, teniendo como tecnología de apoyo, las herramientas del Internet (Web 2.0).

4. La garantía a los ciudadanos, por parte del gobierno, de los derechos de libertad.

5. Partidos políticos con ética política y pública

6. Impulso y creación de instituciones participativas que completen y complementen a las instituciones de la representación clásica liberal. 


\section{La poliarquía plus: base del parlamento abierto}

Hoy estamos en presencia de demandas sociales que exigen ir más allá de las instituciones liberales poliárquicas para que la transparencia genere en las organizaciones una tendencia a hacer frente a las demandas de la divulgación de información y la participación y, la co-creación, y así tener un efecto positivo en la rendición de cuentas. No partimos de una crítica disruptiva de la democracia, por el contrario, creemos que ésta necesita complementarse y completarse con nuevas instituciones desde la sociedad. Estas instituciones serían participativas y colaborativas que permiten activar las herramientas democráticas como el compromiso cívico, inteligencia cívica, control social y colaboración, con la idea de explicar la dinámica de la democracia más allá de la representación y como procedimiento.

En tal sentido, nos hemos guiados por las siguientes dos premisas. Premisa A.- Con la democracia sustentada solamente en las instituciones liberales de la poliarquía, se genera un tipo de transparencia opaca caracterizada por las normas de difusión de información oficial que no revelan cómo se desempeñan las instituciones en la práctica, ya sea en términos de cómo se toman decisiones o de cuáles son los resultados de sus acciones, se concibe políticamente vinculado a los derechos de acceso a la información donde los actores interesados (Organismos garantes, Sujetos obligados y usuarios) no alcanzan tener estrategias de cambio constructivo para la institución, por lo que no son actores reactivos, sino pasivos. En este dirección, este tipo de transparencia va a estar conectada con los valores de la rendición de cuentas liberal caracterizada por los principios del 'buen gobierno' que requiere de políticas que se desarrollen en un ambiente y contexto no hostil, tranquilo y reflexivo que permita revisar todos los aspectos de un problema sin pasar constantemente información al público; segundo, es importante saber que la neutralidad de los funcionarios públicos y su capacidad para ofrecer ayuda y asesoramiento a sus jefes (Secretarios o Ministros) estaría en peligro sí la información sobre esta ayuda o asesoramiento se hace público; y tercero, el proceso de negociación con los diversos grupos de interés podría ser difícil, por lo que no puede ser sensato dar primeramente una posición de negociación. Además, las políticas pueden tener una arista sensiblemente difícil a revelarse; los gobiernos son electos para gobernar y el lugar apropiado para que el gobierno rinda cuentas de las políticas públicas es el poder legislativo (Hunt, 2007). Y en el plano de la participación, el único espacio donde ésta podría generarse es a través del voto.

Premisa B.- Una democracia con poliarquía plus genera un tipo de transparencia clara caracterizada por aquellos programas que dan a conocer información confiable y accesible sobre el desempeño institucional, precisando las responsabilidades de sus funcionarios, particularmente en lo relativo a la toma de decisiones, así como el destino real de sus recursos; se vincula con la rendición de cuentas a través del siguiente itinerario:

- acceso a la información

- calidad de la información

- Transparencia colaborativa

- Rendición de cuentas

Se concibe como derecho, pero además como solución instrumental a problemas de legitimidad y confianza institucional, y expresa una capacidad institucional de tal modo que permite que los actores interesados puedan anticipar estrategias de cambio constructivo para la institución (compromiso cívico). Los actores son proactivos. Este tipo de transparencia va a estar conectada con los valores republicanos de la vigilancia cívica a través de instituciones participativas y colaborativas (rendición de cuentas societal, control social, etc). Así entonces, concebida como plataforma democrática participativa, la poliarquía plus o pospoliarquía (como en adelante la identificaremos) abre posibilidad a la transparencia efectiva, la colaboración, la participación, la rendición de cuentas y la co-creación de lo público. 


\section{$\underline{\text { Para precisar la Poliarquía }}$}

Tal como lo precisara Robert Dahl $(1993,2002)$, partimos del concepto de poliarquía como una democracia ideal, caracterizada por la participación efectiva, igualdad de votos, electorado informado, control ciudadano del programa de acción, inclusión y derechos fundamentales. Estas características delinean lo que para la poliarquía es un proceso democrático visto como un sistema de derechos, es decir, el demos debe tener la oportunidad de cumplir en su comunidad política, con acciones como dar a conocer a los demás miembros sus opiniones de la política adoptada o rechazada; de votar a favor o en contra de una política, partiendo de que todos los votos se computan por igual; de aprender acerca de la política y sobre las posibles políticas alternativas y sus consecuencias probables; de decidir qué asuntos se incluyen en la agenda de la toma de decisiones y cómo han de incluirse allí; de participar en la comunidad política en cualquier momento; y del ejercicio de los derechos ciudadanos. Este proceso democrático debe ser sustentado por un ideal democrático representativo a través de la principal institución que es la de la representación, mediante la cual todas las decisiones y las políticas de gobierno importantes son formuladas por funcionarios electos y que rinden cuenta al electorado vía el voto. Otras instituciones que sustentan a la representación son los procesos eleccionarios libres, limpios y periódicos; la libertad de expresión; la libertad de asociación y las fuentes de información independientes.

En su última versión de la poliarquía (III), Dahl (1993), precisa que "sí el proceso democrático no está anclado firmemente en los juicios del demos, el sistema seguirá deslizándose hacia el quasi-tutelaje" (1993:406), en el que los políticos y representantes son imposibles de controlarlos * por lo que esta etapa sería el resultado de acotar la brecha que separa del demos a las élites de la política pública. Pero para Dahl esta separación se logra gracias a una "masa crítica" de ciudadanos bien informados que complementarían a los organismos legislativos y suplementaría a las instituciones de la poliarquía antes mencionadas.

En este sentido, precisar hoy el rol de una política democrática desde el gobierno nos lleva a inscribir el análisis en el marco de la democratización entendida como poliarquía plus, más allá del régimen (Vargas Cullell, 2008), cuya característica es ver el reconocimiento, la tutela y el ejercicio de los derechos de ciudadanía que tengan como objetivo la inclusión social, política y cultural de los ciudadanos, sobre todo en esta era de migraciones globalizadas en que se ve la necesidad de sociedades multiculturales y plurales aún con conflictos irreconciliables. Ello puede expresarse en términos de eficacia a través de una Constitución y leyes que reconozcan derechos a todas las personas que viven en un territorio y, que prevean los medios para tutelarlos y establezcan los principios y mecanismos para sujetar las autoridades legítimamente constituidas al imperio de la ley.

Además de estas consideraciones, la pospoliarquía es una condición democrática que implica generar instituciones que complementen y completen la soberanía popular y las instituciones de representación clásica. Estas están identificadas con lo siguiente (Vease Cuadro No.1):

La innovación pública y sus instituciones de la co-creación tales como las del debate público en la construcción de agendas públicas y gubernamentales, las oportunidades institucionalizadas de participación y colaboración ciudadana en la formulación, implementación y evaluación de las políticas públicas.

Las de vigilancia a las autoridades gubernamentales: reglas y mecanismos de accountability vertical no electorales que permitan a la ciudadanía canalizar demandas e intereses y a las autoridades y funcionarios tener capacidad de anticipar y corregir acciones públicas que violen sistemáticamente los derechos de las personas).

Las instituciones para las decisiones públicas y las de colaboración. 
Cuadro 1

INSTITUCIONES DE LA POLIARQUÍA PLUS (PODER CIUDADANO)

\begin{tabular}{|c|c|c|c|c|c|}
\hline \multirow{2}{*}{$\begin{array}{l}\text { SABERES } \\
\text { CÍVICOS }\end{array}$} & \multirow{2}{*}{$\begin{array}{l}\text { Acceso al } \\
\text { Poder } \\
\text { Inclusión } \\
\text { ciudadana vía } \\
\text { Poder } \\
\text { Electoral }\end{array}$} & \multicolumn{2}{|r|}{ E j e r c i c i o } & \multicolumn{2}{|c|}{ Poder } \\
\hline & & $\begin{array}{l}\text { Instituciones } \\
\text { participativas en } \\
\text { las políticas } \\
\text { Públicas }\end{array}$ & $\begin{array}{l}\text { Instituciones de } \\
\text { Vigilancia }\end{array}$ & $\begin{array}{l}\text { Instituciones } \\
\text { para la toma de } \\
\text { decisiones }\end{array}$ & $\begin{array}{l}\text { Instituciones de } \\
\text { gobernanza }\end{array}$ \\
\hline $\begin{array}{l}\text { Compromiso } \\
\text { Cívico }\end{array}$ & $\begin{array}{l}\text { Ejercicio del } \\
\text { derecho político. } \\
\text { Inclusión. } \\
\text { Comunicación } \\
\text { abierta }\end{array}$ & $\begin{array}{l}\text { Instituciones de } \\
\text { debate público. } \\
\text { Empoderamiento. } \\
\text { Inclusión. }\end{array}$ & $\begin{array}{l}\text { Inclusión. } \\
\text { Empoderamiento } \\
\text { para la rendición } \\
\text { de cuentas y } \\
\text { Transparencia. }\end{array}$ & $\begin{array}{l}\text { Inclusión e } \\
\text { interacción activa } \\
\text { de los ciudadanos } \\
\text { en los modelos de } \\
\text { toma de } \\
\text { decisiones } \\
\text { Públicas. }\end{array}$ & $\begin{array}{l}\text { Inclusión. } \\
\text { Compromiso de } \\
\text { actores } \\
\text { gubernamentales y } \\
\text { no } \\
\text { Gubernamentales. } \\
\text { Confianza. }\end{array}$ \\
\hline $\begin{array}{l}\text { Inteligencia } \\
\text { Cívica }\end{array}$ & $\begin{array}{l}\text { Fortalecimiento } \\
\text { de lo público. } \\
\text { Inteligencia } \\
\text { colectiva. }\end{array}$ & $\begin{array}{l}\text { Crowdsourcing. } \\
\text { Innovación social } \\
\text { para construir } \\
\text { agendas } \\
\text { Públicas. } \\
\text { Capacidad } \\
\text { institucional de las } \\
\text { organizaciones de la } \\
\text { sociedad civil. }\end{array}$ & $\begin{array}{l}\text { Nuevas } \\
\text { instituciones para } \\
\text { el monitoreo } \\
\text { Colectivo. }\end{array}$ & $\begin{array}{l}\text { Instituciones } \\
\text { para las } \\
\text { decisiones } \\
\text { públicas con } \\
\text { inclusión } \\
\text { ciudadana. }\end{array}$ & $\begin{array}{l}\text { Nueva generación de } \\
\text { conocimiento público. } \\
\text { Confianza. } \\
\text { Capital social. } \\
\text { Crowdsourcing. }\end{array}$ \\
\hline $\begin{array}{l}\text { Control } \\
\text { Social }\end{array}$ & $\begin{array}{l}\text { Inclusión para } \\
\text { la } \\
\text { vigilancia de la } \\
\text { participación } \\
\text { ciudadana. } \\
\text { Vigilancia de los } \\
\text { Procesos } \\
\text { electorales }\end{array}$ & $\begin{array}{l}\text { Mecanismos } \\
\text { institucionales para } \\
\text { la participación y la } \\
\text { colaboración pública. }\end{array}$ & $\begin{array}{l}\text { Monitoreo y } \\
\text { evaluación } \\
\text { Bottom-Up de las } \\
\text { políticas públicas. }\end{array}$ & $\begin{array}{l}\text { Derecho a la } \\
\text { transparencia y } \\
\text { al } \\
\text { acceso a la } \\
\text { información } \\
\text { pública. }\end{array}$ & $\begin{array}{l}\text { Rendición de cuentas } \\
\text { social. }\end{array}$ \\
\hline Colaboración & $\begin{array}{l}\text { Participación en } \\
\text { los procesos } \\
\text { electorales. } \\
\text { Colaboración } \\
\text { cívica } \\
\text { con las Agencias } \\
\text { electorales. }\end{array}$ & $\begin{array}{l}\text { Esquemas de socios } \\
\text { de } \\
\text { expertise y recursos } \\
\text { entre los ciudadanos, } \\
\text { funcionarios públicos } \\
\text { y sector privado para } \\
\text { la implementación de } \\
\text { las } \\
\text { políticas públicas. }\end{array}$ & $\begin{array}{l}\text { Estándares de } \\
\text { rendición de } \\
\text { cuentas basado } \\
\text { en } \\
\text { múltiples actores } \\
\text { productores de } \\
\text { valor } \\
\text { público. }\end{array}$ & $\begin{array}{l}\text { Co-creación de } \\
\text { decisiones } \\
\text { pública. } \\
\text { Instituciones } \\
\text { democráticas } \\
\text { para la } \\
\text { Colaboración. }\end{array}$ & $\begin{array}{l}\text { Mecanismos } \\
\text { institucionales de la } \\
\text { gobernanza } \\
\text { colaborativa. } \\
\text { Intercambio de } \\
\text { conocimiento y } \\
\text { expertise. }\end{array}$ \\
\hline
\end{tabular}

Fuente: construcción propia

\section{Para fortalecer la pospoliarquía: la relación del poder delegado y el poder no delegado}

Para el fortalecimiento de estas instituciones en la poliarquía plus se requiere visualizar dos tipos de poderes que se encuentran y desencuentran a medida de su ejercicio enmarcado en sus reglas. Nos referimos al poder legítimo delegado (poder desde arriba) y al poder legítimo no delegado (poder desde abajo), sustentado en los derechos de ciudadanía. "Los ciudadanos, la fuente de legitimidad del poder político legítimo, delegan poder en las autoridades públicas para que gobiernen en nombre suyo. Este 
delegar es parcial: el ciudadano-agente retiene una cantidad sustancial de poder porque no pierde sus derechos" (Vargas Cullell, 2012).

Estos dos poderes se ejercen según dos reglas. Una es la que regula la cuestión de quién puede ejercer el poder y cómo puede obtenerlo. La otra refiere a la que regula el ejercicio del poder. En cuanto el acceso al poder político del Poder Delegado hablamos de reglas que normalizan el acceso al poder por parte de quienes tendrán la autoridad legal para ejercerlo, así como las reglas que especifican los modos cómo se sustituyen a los gobernantes. En una democracia, estas regulaciones corresponden básicamente al sistema electoral. Y para el ejercicio del poder existen normas que regulan el ejercicio del poder delegado, ese que ha sido conferido a las autoridades públicas. Normas que aplicadas en periodos no electorales y que comprenden un complejo juego de regulaciones cuidadosamente formuladas que estipula los usos legítimos e ilegítimos del poder y las sanciones por transgredir esas normas, tienen como norte asegurar que los gobiernos electos democráticamente gobiernen democráticamente. Nos referimos a la existencia de un Estado de Derecho.

Al hablar del Poder de los ciudadanos, la dimensión de acceso al poder se caracteriza por normas que regulan el acceso al poder. No delegado, el que los ciudadanos no pierden, por parte de los gobernados. Se refiere a la inclusión ciudadana: quien disfruta los derechos de tener voz en asuntos de interés público, de elegir a los que gobernarán la entidad política o de ser elegidos para puestos de gobierno. Esto no es más que la participación electoral, vía la inclusión ciudadana sustentada en los derechos políticos. Y al referirnos al ejercicio del poder no delegado de parte de los ciudadanos, la cuestión se presenta de cómo las personas participan en la vida pública y esgrimen sus derechos frente a los gobernantes y gobernados. La participación ciudadana expresada en la participación en la gobernanza, en la deliberación, ejecución y evaluación de políticas públicas, se trata de la participación cívica en el debate y la deliberación pública y, la colaboración en la co-creación de decisiones y valores públicos.

La relación de estos dos poderes, delegado y no delegado, expresan cuatro lineamientos que le dan razón a la democracia pospoliarquica: uno, las reglas del juego democrático, que corresponden con los principios inspiradores de las normas fundamentales de competencia y de procedimiento que establecen el "quien" y el "como" de la decisión política; dos, los principios de igualdad y de libertad política; tres, los valores de la tolerancia, de la no violencia, del debate libre; y cuatro, los saberes cívicos como herramientas democráticas que se conectan con las instituciones participativas para el ejercicio de los derechos ciudadanos como los del acceso al poder (inclusión ciudadana vía la participación electoral) y del ejercicio del poder como los mecanismos institucionales de participación cívica en la construcción de las agendas públicas, la hechura de la implementación de las políticas públicas, las instituciones de vigilancia, de toma de decisiones públicas y de gobernanza.

En este sentido, las acciones públicas relacionales y con apertura (políticas públicas y decisiones públicas), no serían posibles sin un impulso de una democracia más allá de la representación (Plus Poliarquía). Precisando entonces, podríamos afirmar que la comprensión de la política pública en un contexto de gobernanza más allá de los grupos de presión con el gobierno, nos lleva a detectar instituciones participativas en los espacios públicos de deliberación, debate y crítica, así como instituciones colaborativas para la co-creación de lo público. El espacio público lo veríamos entonces como la gama heterogénea y diversa de posibilidades, de visiones, de creencias, de percepciones, de problemáticas y de soluciones donde el diálogo y la deliberación permiten sistematizar en forma legítima acuerdos, consensos y disensos para la toma de decisiones públicas. Es compartir para co-crear.

De esta manera, las instituciones de debate y participación abren caminos a la democracia para que los gobiernos escuchen las voces de los ciudadanos, así como los diferentes tipos de diálogos. Avritzer (2010) tomando el caso de Brasil, las denomina instituciones democráticas participativas y las caracteriza:

1) Primeramente, como híbridos entre la participación y la representación, así como los actores de la sociedad civil y los del Estado. Los actores del Estado mantienen la soberanía formal sobre los procesos de toma de decisiones en algunos ámbitos como la salud y la planeación urbana, transfiriendo esta soberanía a una instancia más amplia. Sin constituir un poder dual, las organizaciones de la sociedad civil toman en serio las acciones de los actores del Estado al interactuar con ellos. 
2) La segunda característica de las instituciones participativas es que dentro de éstas las organizaciones de la sociedad civil transforman las prácticas anteriores de organización social en una forma permanente de organización política con mandatos establecidos, es la institucionalización de la participación ciudadana.

3) La tercera característica tiene que ver con el nuevo papel de la sociedad política, por ejemplo los partidos políticos y los actores de la sociedad civil desempeñan roles clave en las instituciones participativas en el momento en que vinculan las ideas de participación de los ciudadanos con los actores de la sociedad política.

4) Y la última característica mencionada tiene que ver con el diseño institucional innovador, es decir, cómo las instituciones nuevas participativas detonan la innovación y las relaciones horizontales.

En este contexto en consecuencia, innovar la democracia va de la mano con el fortalecimiento tanto de las instituciones participativas democráticas (Rizvi, 2008), como de las relaciones societales y de éstas con el gobierno. "Hay innovación cuando ciertos tipos de problemas no tienen solución en las instituciones existentes. Los actores deben conseguir soluciones innovadoras de sus problemas dado a la insuficiencia institucional" (Harrison y Clain, 2007:8).

Pero estas características identificadas llevan a la reflexión considerar los efectos de las instituciones participativas subordinados al proceso formativo de la ley en el contexto de un sistema de democracia representativa y no entregar los objetivos y resultados de la participación al jefe de Estado o Presidente, Gobernador o Alcalde para dirimir conflictos con otros poderes constitucionales (Soto Barrientos, 2013).

Estas instituciones participativas también ofrecen un moderno interfaz para restablecer la plaza pública (public town square), con el objeto de crear espacios para que las distintas voces no sólo sean escuchadas y conectadas entre ellas, sino empoderadas. La poliarquía plus puede actuar como una política democrática creadora para abrir el acceso de los datos e información y facilitar nuevas oportunidades de acuerdos para añadir valor y co-crear las políticas y los servicios públicos. En consecuencia, los procesos de transparencia y rendición de cuentas facilitan al ciudadano su interacción y colaboración con el gobierno, además de fortalecer el control social, la inteligencia cívica y el compromiso cívico.

También consideramos que para el fortalecimiento de las instituciones participativas pospoliarquica, se requiere de una plataforma institucional para la innovación y la co-creación. En este sentido, las redes sociales y las herramientas Web 2.0 son puntos fundamentales en la nueva ruta democrática, ya que facilita la interacción del gobierno con los ciudadanos, creando nuevos caminos en el cual el debate público puede producirse. Coleman (2009) lo denomina como un proceso revolucionario porque es activo y rápido. En este sentido, este autor parte de la premisa siguiente: "cuando las personas que ahora están siendo escuchadas, van a hablar, y hablan, ofrecen ideas que nunca se les pudo ocurrir al gobierno". Precisando, podríamos decir que éste sirve mejor a la gente cuando decide basado en decisiones de la gente.

"Es un proceso colaborativo y de co-creación sustentado en la participación de los individuos en asociaciones independientes que usan tecnologías de información para alcanzar resultados compartidos, impactando el lugar de trabajo, las comunidades, las democracias locales, nacionales y la economía, así como la obtención de beneficios sociales, tales como la transparencia y la rendición de cuentas en los gobiernos" (Edelmann et al., 2012: 23).

\section{La herramienta democrática de los saberes cívicos en la poliarquía plus}

Hemos venido afirmando que la política democrática pospoliárquica parte del poder de los ciudadanos en su relación con el Poder delegado del Estado. Por esta razón, concebimos primeramente esta 
política como el reconocimiento de la creatividad y el resultado que imprimen los ciudadanos y actores no gubernamentales en su relación con los actores políticos y gubernamentales. Es decir, la participación de los ciudadanos es fundamental en esta nueva visión de la poliarquía ya que tiene como base a las instituciones participativas creadas como un proceso de democratización. En esta dirección, presentamos también a la poliarquía plus como un proceso de innovación pública institucional atendiendo a una premisa y a dos direcciones. La premisa es que no puede haber innovación institucional democrática sin un sector público, unas instituciones políticas y una ciudadanía innovadores. En cuanto a las direcciones, la primera de ellas no es más que los arreglos de los ciudadanos, actores políticos y gubernamentales y no gubernamentales que permiten identificar problemáticas públicas, así como herramientas y dispositivos novedosos de co-creación para el logro de respuestas y soluciones nuevas en el espacio de lo público presencial y digital. Y la otra dirección, consecuencia de la anterior, se refiere a las modalidades democráticas de articulación de estos actores en la organización pública, produciendo esto una nueva configuración entre el Estado y la sociedad que asegura el aprendizaje colectivo necesario para la cocreación de lo público. Solo así vemos a los gobiernos más relacionales, a la gestión pública más participativa y a las políticas públicas, en todo su ciclo, más deliberativas en la construcción de la agenda, más incluyentes en aportar soluciones (diseño), y más colaborativas por los múltiples actores en acción en la implementación.

Partiendo de este concepto, precisamos que el centro del análisis aquí son los componentes de los saberes cívicos de los ciudadanos, expresado en que el trabajo contenido en aquellos es hecho tomando como sustento el compromiso cívico de los ciudadanos con el gobierno y con la sociedad, así como el compromiso de los actores del Estado con los ciudadanos y con ellos mismos, facilitando la inteligencia cívica, el mejoramiento del control de la gestión vía la contraloría social, así como una concepción compartida de lo público (competencias de colaboración) para el logro legitimado de resultados públicos.

Definimos estos saberes cívicos como todo lo que es y lo que sabemos de la convivencia democrática, de los derechos y obligaciones como ciudadanos, así como de las estructuras, funciones y medios del poder del Estado y del Gobierno. Implica la construcción propia del conocimiento ciudadano pertinente al grado de su cultura democrática. Es estar dispuesto a participar tanto presencial como a través de las tecnologías de información y comunicación (TCIs) en el discurso racional con la idea de ir generando un tipo de poder no delegado, desde los ciudadanos, en que su ejercicio establezca la cuestión de cómo las personas participan en la vida pública, esgrimiendo sus derechos frente a los gobernantes para la co-creación de lo público (Mariñez, 2015).

Analizamos a continuación cada uno de los componentes de los saberes cívicos y su relación con las instituciones pospoliárquicas (debate público en la construcción de las agendas, hechura e implementación de las políticas públicas, vigilancia, toma de decisiones y colaboración).

\section{A.- El compromiso cívico}

El primer componente es el compromiso cívico. Se entiende éste como la forma en que los ciudadanos, a través de una obligación o promesa, logran impulsar el asociacionismo con la idea central de llevar a cabo el debate, la deliberación y la solución de problemas públicos. Se trata de un punto más allá de la participación ciudadana que asegura que toda la gente haga uso de sus derechos de libertad, ya que estos y la ciudadanía, según Barber (2004), son correlativas, cada una sostiene y da vida a la otra. No importa la seguridad, los derechos individuales y la autonomía que disfruten: los hombres y mujeres que no se hacen directamente responsables de las políticas que determinan sus vidas a través de la deliberación, la decisión y la acción comunes no son en absoluto libres. En este orden de ideas, Subirats (2015), nos precisa que "los debates sobre la gestión de recursos, la gestión de servicios, el gobierno y la gestión de las instituciones, toman otro sentido si se parte de las siguientes premisas: construir una nueva política local desde los ciudadanos, desde los barrios, desde los expertos y saberes ciudadanos, desde los políticos-ciudadanos, desde los que saben por qué y han padecido y luchado contra los efectos de las decisiones que han ido tomando los que aseguraban que sabían lo que hacían y que lo hacían por nuestro bien" (Subirats, 2015;3). Es por ello que el "compromiso cívico y los formatos participativos que incluye los actores de la sociedad civil, es la más importante dimensión de la renovación institucional de la representación" (Avritzer, 2012:2). 
Nos referimos entonces a las diversas formas de diálogo ciudadano que son altamente inclusivas y a la deliberación para la hechura de las políticas y decisiones públicas. De esta manera el mismo Avritzer puntualiza claramente dos dimensiones de la democracia deliberativa, una es la dimensión pública que ayuda a los debates públicos informales -muy escaso en los partidos políticos-; y una segunda dimensión en el que el proceso de mejoramiento de la democracia es la búsqueda de nuevos formatos de instituciones que pueden mejorar las prácticas que usualmente se desarrollan en las democracias poliárquicas (Avritzer; 2012). Así, "la clave de la virtud deliberativa como la reciprocidad, quiere decir, argumentar en términos que otros pueden aceptar" (Gutmann y Thomson, 1996, citado por Dryzek, 2009). La inclusión es un aspecto importante cuando hablamos del compromiso cívico, ya que tiene como objetivo proporcionar a las personas un sentido de dirección para que las actividades asociativas y comunitarias tengan voz en su relación con el gobierno y así ayudarle a mejorar sus decisiones. El éxito del compromiso cívico con el gobierno requiere de innovación (Fung; 2008).

Así pues, el compromiso cívico ciudadano le da soporte efectivo al funcionamiento de la democracia, al gobierno y a la administración pública, generando contextos para la legitimidad de la acción gubernamental, el éxito de la implementación de la política pública y el logro de resultados sociales: valor social y valor público.

La visualización de King y Martinelli (2005), es pertinente ya que el compromiso de los ciudadanos, los actores gubernamentales y los no gubernamentales y, a nivel organizativo en sus asociaciones en la vida pública es una condición para una plataforma de democratización. Así, afirman que el empoderamiento y el compromiso de los ciudadanos deben ir de la mano de las siguientes interfaces:

- El interfaz entre el Político y los Ciudadanos (político) tales como la efectividad de las técnicas y las herramientas.

- El interfaz Ciudadano-Burocracia (Administración) tales como el compromiso ciudadano y el empoderamiento en el desarrollo de las políticas públicas y en el establecimiento de prioridades.

- La rendición de cuentas gubernamental, tales como el compromiso ciudadano mejorando los rendimientos del gobierno, así como la rendición de cuentas para mejorar los procesos y resultados

También Bailur y Gigler (2014), parten de que el compromiso cívico implica el empoderamiento ciudadano, por la cual se requiere de los siguientes tres recursos: la participación, la transparencia y la rendición de cuentas. Por un lado, la participación les hace elevar sus requerimientos y voces (con la idea de que sean escuchados). En este sentido, las herramientas Web 2.0 proveen una oportunidad para empoderar a los ciudadanos porque bajan los impedimentos para participar. Con estas herramientas estos pueden acceder directamente a la información y comunicación en lugar de ser dependientes de actores intermediarios. Por otro lado, el recurso de la transparencia es importante para el empoderamiento porque se trata de cualquier intento (por el gobierno o ciudadanos) de colocar información o proceso que antes eran opacos en el dominio público para el uso de los grupos ciudadanos, proveedores o hacedores de política. Y el tercer recurso del empoderamiento es la rendición de cuentas (accountability), entendida como la relación entre el poder titular (proveedor titular) y el poder delegado (los demandantes). Se requieren normas establecidas para adquirir información sobre acciones y su pertinencia, hechura de las decisiones, y la identificación y sanción de resultados insatisfechos.

En vista de lo planteado, el compromiso cívico lo podemos visualizar en base a tres elementos: uno, el conocimiento político de la Constitución de un País y las leyes, de las instituciones políticas, de la estructura gubernamental y estatal, así como de los actores políticos y sociales. El segundo elemento es el interés político tanto de los actores gubernamentales como de los ciudadanos, expresado en la participación cívica, la colaboración y la co-creación; y el último elemento lo relacionamos con la discusión política en todos los órdenes de la vida cívica y en todos los poderes. 


\section{B.- La inteligencia cívica}

El otro componente de los saberes cívicos es la inteligencia cívica, vista como inteligencia colectiva directa, dirigida a enfrentar los desafíos compartidos $\dagger$. Es decir, dirigir las capacidades percibidas, actuales y potenciales de los individuos, grupos y organizaciones sociales, para responder inteligente, efectiva y equitativamente a los desafíos actuales para el logro del desarrollo de la convivencia democrática. La inteligencia cívica no es más que tener información y comprenderla para compartirla a quienes necesitan respuestas. Visto así Shuler (2008) afirma que la inteligencia cívica se centra en el rol de la sociedad civil y lo público, y su carácter es participativo y colaborativo, de allí lo cívico de este tipo de inteligencia.

En otras palabras, implica conocer, responder y proveer, por lo que se podría entender como la capacidad de las organizaciones ciudadanas y comunitarias de adquirir y aplicar conocimientos; o bien, como la capacidad para adaptar efectivamente, o simplemente para la resolución de problemas, aprendizaje, razonamiento, predicción, reflexión e imaginación de los ciudadanos (La Duke, 2008).

Inteligencia cívica no es más que la lógica de la co-creación de soluciones, de definición de problemas sociales y de colaboración. Por eso el Crowdsourcing juega un papel importante en la inteligencia cívica ya que los ciudadanos aprovechan el Internet para adquirir recursos, conocimiento, experiencia o tiempo (Colaboración abierta). Zhao y Zhu (2012, citado por Seltzer y Mahmoudi; 2012) definen el crowdsourcing como un sistema de inteligencia colectiva. Y Surowieck (2005), habla de la sabiduría del público, "en las circunstancias adecuadas, los grupos son muy inteligentes, y con frecuencia son más inteligentes que las personas más inteligentes del grupo" (Surowiecki; 2005: xiii; citado por Seltzer y Mahmoudi; 2012). En muchos países no desarrollados, el crowdsourcing es aplicable en el marco de consultas populares, monitoreo de elecciones, procesos de redacción de Constitución, o en cualquier lugar que se asegura de que las voces de la diversidad étnica y política y, grupos minoritarios serán escuchados (Bott, Sigler; Young; 2014).

\section{C.- El control social}

El tercer componente de los saberes cívicos es el control social, que no es mas que la participación ciudadana orientada al monitoreo, la vigilancia y la evaluación por parte de personas u organizaciones sobre programas y acciones gubernamentales, incidiendo directamente en la esfera pública ya sea formalmente por medio de su inclusión en comités de obras, órganos colegiados, etc., o indirectamente por medio de la activación de los sistemas de quejas y denuncias (Hevia de la Jara, 2006). Lo que sí es cierto es que el control social es un tipo de control que en vez de originarse desde las instituciones administrativas y políticas del Estado emana desde la sociedad, el control directo desde los ciudadanos a los políticos y funcionarios públicos.

Hoy tenemos un nuevo marco de análisis para el control social a partir de las tecnologías Web 2.0. A través del software social se logra una serie de aplicaciones que pretenden ampliar las posibilidades de comunicación y de conexión entre personas que ya permiten el e-mail o los sistemas de mensajería instantánea. Son herramientas colaborativas que une a una serie de personas con el fin de facilitar el flujo de información (agendas, mensajes, denuncias) y la realización de trabajos conjuntos entre ellas (colaboración en el monitoreo o vigilancia de las políticas públicas). Todas las herramientas colaborativas están basadas en Web 2.0 del Internet.

De esta manera, los ciudadanos no solamente tienen acceso a la información, sino que se convierten en participantes con expresión activa. Así, la elección de los usuarios como sus opiniones con respecto a los servicios públicos son fundamentales para la legitimidad del gobierno y la democracia. En consecuencia partimos de cuatro ejes que hacen del control social más efectivo. El Primer eje es la transparencia colaborativa. Debe existir un proyecto de datos abiertos, aplicado como una filosofía y forma de administración, donde todos los datos públicos estén disponibles y actualizados en un formato libre,

\footnotetext{
† "El concepto de inteligencia colectiva se opone a la idea de que el conocimiento legítimo viene desde 'arriba', de la universidad, de la escuela, de los expertos, reconociendo, al contrario, que nadie sabe todo y que cualquiera sabe algo" (Levy, 2004: 7).
} 
gratuito y sin ninguna restricción. El segundo eje se relaciona con la organización y las capacidades de los ciudadanos. Precisar la organización y capacidad ciudadana en el marco de las TCIs para el control social, se requiere que los ciudadanos logren la legitimidad técnica para poder hacer uso de las plataformas software social y sus herramientas de colaboración que permiten comunicar, coordinar y compartir recursos, aplicaciones y contenidos. El tercer eje es el acceso a la información. Se parte de la base de que el acceso a la información es un derecho ciudadano, reconocido en muchos países a nivel constitucional. Y el cuarto eje lo relacionamos con lo público. El control social no sólo se aplica al gobierno, sino a todos los actores que intervienen en la producción del valor público. Saxton y Guo (2011) han venido afirmando en base a sus investigaciones que con la difusión de las tecnologías del internet, aparecen dos dimensiones fundamentales de la rendición de cuentas de las organizaciones civiles: la divulgación y el diálogo. Quiere decir, que si la producción de lo público es colaborativa (co-creación), los diversos actores (ciudadanos, gubernamentales y no gubernamentales) son objeto de ser controlados por sus acciones.

\section{D.- La participación colaborativa}

El cuarto y último componente de los saberes cívicos es la participación colaborativa. Es evidente que para que la participación colaborativa se cristalice y se convierta en un factor de innovación democrática, es menester la existencia de una política gubernamental que posibilite un mayor acercamiento a través del diálogo entre gobierno y ciudadanos, dándosele así cabida a la atención y la gestión de las demandas, a la negociación e interlocución entre ellos, y a la colaboración.

Harrison, Guerrero et al.(2011) afirman que la deliberación y la participación deben dar la oportunidad para el intercambio de visiones con el objeto de determinar el curso de acción, para asegurar que diversos puntos de vista de ciudadanos, grupos civiles y actores no gubernamentales sean incorporados a la acción del gobierno. Es decir, que la participación colaborativa se da en el momento en que los individuos son parte integrante, con su expertise, junto con el gobierno, del proceso de co-creación de las decisiones para generar soluciones que luego serán implementadas.

La participación colaborativa implica un cambio en el juego de las legitimidades políticas, técnicas, sociales y éticas de los ciudadanos y otros actores no gubernamentales, por lo que nos lleva a ver la lógica gubernamental operando con esquemas de co-creación, ya que ésta "envuelve dinámicas innovadoras para encontrar soluciones de una manera poco convencional, ya sea a través del desarrollo de una nueva solución o un nuevo enfoque que incorpora al mismo tiempo, una variedad de actores. Lo distintivo de la innovación en procesos participativos de la co-creación, por lo tanto no se relaciona sólo con una nueva técnica sino con una forma de generar nuevo conocimiento de manera colectiva (Zurbriggen; González, 2014: 339).

La participación colaborativa también activa a la pospoliarquía en el momento en que abre una posibilidad de diálogo, comunicación, legitimidad y confianza, convocando a los diferentes actores de la sociedad a trabajar juntos, reconociendo que los ciudadanos poseen información propuesta por diversos actores que puede ser usada para resolver los problemas públicos. "Un nuevo paradigma de la colaboración en la administración pública requiere que ciertos comportamientos en línea sean aprendidos, comprendidos y adoptados" (Edelmann, Hölchtl and Sachs: 2012: 22).

Podríamos hablar así de la era de la colaboración impulsada por la aparición de nuevos talentos digitales, creación de nuevas estructuras sociales que superan por su complejidad de las anteriores, y por nuevos modos de creación de valor tanto público como social. Esta afirmación nos deja claro lo que Noveck (2010) ha planteado que la colaboración es una forma de participación democrática que es igualitaria pero diferente a la concepción tradicional del término. 


\section{A manera de Conclusión}

Este artículo analizó la relación entre parlamento abierto y gobierno abierto en el contexto de las herramientas democráticas de la poliarquía plus. Asumimos que todos los enfoques de gobierno abierto tienden a identificar, evaluar y caracterizar diferentes formas de integración ciudadana en el sector público tanto por la vía de la transparencia, el acceso a la información y la rendición de cuentas, así como por la colaboración, la participación y la co-creación. En este punto, para los efectos analíticos, desagregamos el concepto de gobierno abierto en dos dimensiones. Una, en su dimensión relacional (participación y colaboración), y la otra en su dimensión de apertura (transparencia, rendición de cuentas y apertura a la información).

En este sentido, pudimos precisar que la esfera pública, asidero de estas dos dimensiones está construida alrededor del sistema de comunicación social y de redes de internet, particularmente en el espacio social de la Web 2.0 (Open data, datos disponibles, Open Collaboration, Open Knowledge, ciudadanía 2.0, free software o social -Facebook, MySpace-, Open Source, Big data, linked data), que genera colaboración, interacción e inteligencia cívica, utilizando las aplicaciones que los usuarios pueden usar para entrar al internet o intranet (webmail, wikis, weblogs, wikis, wikipedia). Ello ha implicado un cambio del proceso, modo y patrón de gobierno, de su relación con la sociedad, del paso de la cooperación a la colaboración y la co-creación, así como del tipo de transparencia colaborativa. Así seis principios clave comprende este concepto de gobierno: transparencia, participación, colaboración, rendición de cuentas, cocreación y TICs.

Por ello, el concepto de gobierno abierto nos remite a un tipo de organización relacional como opuesta a la organización cerrada de estilo weberiana ya que las organizaciones públicas de hoy tienden a caracterizarse por ser abiertas y flexibles, con una visión de red, un liderazgo relacional e innovadoras por el uso de las TICs y también por generar nuevos procesos y organización para un nuevo servicio público.

Estos elementos del gobierno abierto y sus implicaciones lo analizamos como el fundamento contextual para que el Parlamento -institución de la representación- responda a las demandas sociales bien de apertura, bien relacionales- que exigen ir más allá de las instituciones liberales poliárquicas para que la transparencia genere en las organizaciones una tendencia a hacer frente a las demandas de la divulgación de la información, así como de la participación, la colaboración y la co-creación.

Ante la crisis de representación, se han podido identificar cuatro elementos que empujan el déficit democrático. Ellos son, tal como lo afirma Fung: la poca claridad de las preferencias de los ciudadanos; la debilidad institucional de los mecanismos electorales que proporcionan señales débiles a los políticos con respecto a las preferencias de los ciudadanos; el mecanismo de elección insuficiente para propósitos de rendición de cuentas (accountability) de los políticos; y la falla del Estado al no poseer la capacidad técnica para proporcionar resultados razonables de políticas que materializan las preferencias de los ciudadanos.

Por estas insuficiencias, partimos que lo sustantivo de un Parlamento Abierto es establecer una nueva relación entre representantes y representados, en términos de colaboración, rendición de cuentas, acceso a la información, participación ciudadana y uso de tecnologías de información. Ello con la intención de minimizar por una parte, la insatisfacción popular, y por la otra, la desconfianza en la institución parlamentaria. Afirmamos en el transcurso del análisis que la arena política en redes -esfera del debate público-, permite el trabajo colaborativo de diversas formas. Como por ejemplo, individuos pueden monitorear y desvirtuar el poder de los medios convencionales, así como organizar acciones políticas, mediante las diversas formas de acceso a Internet, para que los individuos y grupos estarían más aptos a observar, relatar, comentar y analizar hechos, ejerciendo en suma, las funciones de agentes de medios con capacidad de atraer la atención pública a los diversos asuntos políticos.

Para llevar a cabo esto se requiere generar instituciones más allá de las instituciones liberales poliárquicas. Estas instituciones que serían participativas y colaborativas, permiten activar las herramientas democráticas como el compromiso cívico, inteligencia cívica, control social y colaboración, con la idea de explicar la dinámica de la democracia más allá de la representación y como procedimiento. Así, las instituciones de debate y participación abren caminos a la democracia para que los gobiernos escuchen las voces de los ciudadanos, así como los diferentes tipos de diálogos para la colaboración y co-creación. 
Pudimos proponer la relación entre cada uno de los componentes de los saberes cívicos y su relación con las instituciones pospoliárquicas (debate público en la construcción de las agendas, hechura e implementación de las políticas públicas, vigilancia, toma de decisiones y colaboración).

\section{BIBLIOGRAFÍA}

Alford, J (2009) Public Sector Clients: From Service-Delivery to Co-production. Basingstoke, Palgrave Macmillan.

Avritzer, Leonardo (2012) “Democracy beyond aggregation the Participatory dimension of Public Deliberation”. Journal of Deliberation: Vol.8: Iss 2, Art. 10

Avritzer, Leonardo (2010) Las instituciones participativas en el Brasil democrático.

Biblioteca Universidad Veracruzana. México

Bailur, Savita and Bjorn-Soren Gigler (Editors) (2014) Closing the Feedback Loop. Can Technology Bridge the Accountability Gap? The World Bank. Washington, DC.

Barber, Benjamin (2004) Democracia fuerte. Política participativa para una nueva

época. España: Almuzara.

Bott, Maja; Börn-Soren Sigler; Gregor Young (2014) "The rol of Crowsourcing for better Governance in Fragile State Contexts". In Singler, Börn-Soren and Savita.

Coleman, Casey (2009) Web 2.0 tools encourage Public Debate. WWw.gsa.gov

Colomer, Josep M. (2009) Ciencia de la política. Ariel Ciencia Política, Barcelona.

Cusba, Enrique (2012) "Colaboración: El gobierno en doble vía con las personas, en Gastón Concha y Alejandra Nasser (Coordinadores), El desafío en el gobierno abierto en la hora de la igualdad, Documento de proyecto, Chile: CEPAL.

Dahl, Robert (1993) La democracia y sus críticos. Paidós Editores, España.

Dahl, Robert A. (2002) La poliarquía. Participación y oposición. Editorial Tecnos. España.

Declaración de lanzamiento de la Alianza para el Parlamento Abierto en México (2014). http://www.senado.gob.mx/comisiones/cogati/eventos/docs/Declaracion_220914.df

Dryzek, John S. (2009) "Democratization as Deliberative Capacity Building". Comparative Political Studies. Vol. 42, Number 11, pp. 1379-1402.

Du Gay, Paul (2000) En elogio de la burocracia. Weber-Organización-Ética. Siglo XXI de España Editores.

Edelmann, Noella, Johann Höchtl and Michael Sachs (2012) "Collaboration for Open Innovation. Process in Public Administration". In Y. Charalabidis and S. Koussouris (eds) Empowering Open and Collaboration Governance. Springer-Verlag. Berlin Heidelberg.

Fung, Archon (2008) "Citizen Participation in Government Innovations" in Sandford Borins Innovations in government. Research, Recognition, and Replication. Harvard University and Brookings Institute Press.

Fung, Archon (2007) "Democratic Theory and Political Science: A Pragmatic Method of Constructive Engagement", American Political Science Review Vol. 101, No. 3 (August 2007): 443-58.

Harrison, Teresa M, Santiago Guerrero, G. Brian Burk and Meghan Cook (2011) "Open Government and EGovernment: Democratic Challenges from a Public Value Perspective", en The Proceeding of the 12th Annual International Digital Governement Research Conference.

Harrison, Denis et Juan-Luis Klein (2007) "Introduction", en Denis Harrison y Juan-Luis Klein (Coordinadores) L’Innovation sociale. Émergence et effets sur la transformation des societies. Presses de l'Université du Québec. 
Hevia de la Jara, F. (2006). La Contraloría Social mexicana: Participación ciudadana para la rendición de cuentas. Diagnóstico actualizado a 2004. México: Centro de Investigaciones y Estudios Superiores en Antropología Social (CIESAS)/Instituto de Investigaciones Histórico-Sociales, Universidad Veracruzana.

Hilgers, Denis and Christoph Ihl (2010) "Citizensourcing: Appliying the Concept of Open Innovation to Public Sector". The International Journal of Public Participation. Vol. 4, No. 1.

Hunt, Mikeal (2007) "Secrecy, Privacy and Freedom of Information". Training Public Administration. Vol. 27, No. 2. Pp. 25-36

Holzner, B., \& Holzner, L. (2006). Transparency in global change: The vanguard of the open society. Pittsburgh, PA: University of Pittsburgh Press.

Grimmelikhijsen, Stephen (2012) "Linking Transparency, Knowledge and citizen Trust in Governement: An Experiment". International Review of Administrative Sciencies. 78 (1), 50-73. Sage Publications.

Kickley, M (2008) The Grounds for Citizen Engagement and The Roles of Planners. Saarbruecken. VDM.

King, Simrell Cheryl and Alicia Seegers Martinelli (2005) "Innovation in Citizen Engagement and Empowerment Beyond Boundaries" The Innovation Journal: The public Sector Innovation Journal, Vol 10 (1), Paper 1.

LaDuke, Bruce (2008) "Knowledge creation in collective intelligence", en Mark Tovey (Editor) Collective Intelligence: Creating a prosperous World at Peace., Earth Intelligence Network (EIN). http://www.scip.org/files/resources/toveycollective-intelligence.pdf (Consultado el día 10 de marzo del 2016)

Lévy, P., (2004) Inteligencia colectiva por una antropología del ciberespacio. Organización Panamericana de la Salud (Unidad de Promoción y Desarrollo de la Investigación y el Centro Latinoamericano y del Caribe de Información en Ciencias de la Salud), Washington, 2004.

Mariñez Navarro, Freddy (2015) Innovación Pública. Para que funcionarios y ciudadanos actúen con saberes cívicos. FONTARAMA editores. México.

McGuire (2006) "Collaborative Public Management: Assessing What We Know and How We Know It". Public Administration Review. 66 (1), 33-43.

Noveck, Beth Simone (2010) "The Single Point of Failure" in Lathrop, Daniel \& Laurel Ruma (Edited by) Open Government. Collaboration, Transparency, and Participation in Practice. O’Reilly, USA, First Edition.

Noveck, Beth S. (2009) Wiki Government: How Technology Can Make Government Better, Democracy Stronger, and Citizens More Powerful . Brookings Institute Press: Washington DC

Obama, B (2009) Transparency and Open Government. http://www.whitehouse.gov/the_press_office/Transparency and Open Government/

OECD (2004) Promise and Problems of E-Democracy: Challenge of Online Citizen Engagement. Parías: OECD Publications

O’Donnell, Guillermo (1994) "Delegative Democracy”. Journal of Democracy, Vol.5, No.1. pp. 55-69

O'Leary, Rosemary and Nidhi Vij (2012) "Collaborative Public Management : Where Have We Been and Where Are We Going?" en The American Review of Public Administration 42(5).

Oszlak, Oscar (2012) "Gobierno abierto: promesas, supuestos, desafíos". VIII Conferencia Anual INPAE: Gobierno Abierto. Por una gestión pública, más transparente, participativa y colaborativa. San Juan, Puerto Rico. ¿Qué es la Alianza para el Parlamento Abierto? http://www.parlamentoabierto.mx/

Raelin, Joseph A. (2011) “The End of Managerial Control?” Group \& Organization Management , 36(2): 135-160

Rainey, Hall G. (2003) Understanding and Managing Public Organization, 3tr Ed. USA, Jossey-Bass publishers.

Rizvi, Gowher (2008) "Innovation in Government: Serving Citizens and Strenthening Democracy". In Sanford Borins Innovations in government. Research, recognition, and replication. Ash Institute for Democratic Governance and Innvovation. Jonh F. Kennedy School of Government, Harvard University.

Setzer Ethian; Dillon Mahmoudi (2012) "Citizen Participation, Open Innovation, and Crowdsourcing: Challeges and Opportunities for Planning". Journal of Planning Literature. 28(1). 3-18

Shuler, Douglas (2008) "Civic intelligence and the public sphere". In Mark Tovey Collective Intelligence: Creating a Prosperous World at Peace. The Wealth of Networks: Remixed Highlights. Earth Intelligence Network, USA.

Soto Barrientos, Francisco (2013) El desafío de la participación. Referendo e iniciativa legislativa popular en América Latina y Europa. LOM Editores, Chile. 
Subirats, Joan (2015) "Modernidad y protagonismo ciudadano”. El País (abril 5). www.elpais.com

Vargas Cullell, Jorge (2012) "La calidad de la democracia y el estudio comparado de la democratización" en Gerardo Munck y Sebastián Mantilla La calidad de la democracia: perspectivas desde América Latina. http://www.hss.de/fileadmin/americalatina/Ecuador/downloads/Version_1_Libro _Calidad_de_la_democracia_1.pdf (Consultada el día 13 de marzo de 2016)

Vargas Cullell, Jorge (2008) "Democratización y calidad de la democracia". En Oscar Ochoa (coordinador) La reforma del Estado y la calidad de la democracia en México. Una reflexión sobre sus instituciones. Miguel Angel PorrúaEGAP, México, 2008.

Weber, Max (2013) ¿Qué es la burocracia? www.scribd.com/doc/145924959/Libros-Tauro-t (Consultado el 7 de abril 2016)

Zurbriggen, Cristina y Mariana González Lago (2014) "Innovación y Co-creación. Nuevos desafíos para las políticas públicas”. Revista de Gestión Pública, Vol. III, No. 2. 